\title{
Star-formation efficiency and metal enrichment of the intracluster medium in local massive clusters of galaxies (Corrigendum)
}

\author{
Y.-Y. Zhang ${ }^{1,2}$, T. F. Laganá ${ }^{3,1}$, D. Pierini ${ }^{4, \star}$, E. Puchwein ${ }^{5,6}$, P. Schneider ${ }^{1}$, and T. H. Reiprich ${ }^{1}$ \\ 1 Argelander-Institut für Astronomie, Universität Bonn, Auf dem Hügel 71, 53121 Bonn, Germany \\ e-mail: yyzhang@astro.uni-bonn.de \\ 2 National Astronomical Observatories, Chinese Academy of Sciences, 100012 Beijing, PR China \\ 3 Universidade de São Paulo, Instituto de Astronomia, Geofísica e Ciências Atmosféricas, Departamento de Astronomia, \\ Rua do Matão 1226, Cidade Universitária, CEP: 05508-090, São Paulo, SP, Brasil \\ ${ }^{4}$ Max-Planck-Institut für extraterrestrische Physik, Giessenbachstraße, 85748 Garching, Germany \\ 5 Heidelberger Institut für Theoretische Studien, Schloss-Wolfsbrunnenweg 35, 69118 Heidelberg, Germany \\ ${ }^{6}$ Max-Planck-Institut für Astrophysik, Karl-Schwarzschild-Straße 1, 85741 Garching, Germany
}

A\&A, 535, A78 (2011), DOI: 10.1051/0004-6361/201116803

Key words. cosmology: observations - galaxies: clusters: general - methods: data analysis - surveys - X-rays: galaxies: clusters errata, addenda

Table 1. Properties of the 19 galaxy clusters.

\begin{tabular}{|c|c|c|c|c|c|c|c|c|}
\hline \multirow[t]{2}{*}{ Name } & \multicolumn{2}{|c|}{ X-ray center $(\mathrm{J} 2000)$} & \multirow[t]{2}{*}{ Redshift } & \multirow{2}{*}{$\begin{array}{c}M_{500} \\
10^{14} M_{\odot} \\
\end{array}$} & \multirow{2}{*}{$\begin{array}{c}M_{500, M-M_{\text {gas }}} \\
10^{14} M_{\odot} \\
\end{array}$} & \multirow{2}{*}{$\begin{array}{c}M_{\text {gas, } 500} \\
10^{13} M_{\odot}\end{array}$} & \multirow{2}{*}{$\begin{array}{c}M_{*, 500} \\
10^{12} M_{\odot}\end{array}$} & \multirow{2}{*}{$\begin{array}{l}\text { Undisturbed } \\
\text { /cool core }\end{array}$} \\
\hline & $\mathrm{RA}$ & Dec & & & & & & \\
\hline A0085 & $00: 41: 50.306$ & $-09: 18: 11.11$ & 0.0556 & $6.37 \pm 1.00$ & $5.68 \pm 0.37$ & $8.13 \pm 0.38$ & $7.36 \pm 1.00$ & $\mathrm{Y} / \mathrm{S}$ \\
\hline A0400 & $02: 57: 41.349$ & $+06: 01: 36.93$ & 0.0240 & $1.83 \pm 0.39$ & $1.07 \pm 0.07$ & $1.36 \pm 0.05$ & $4.39 \pm 1.06$ & $\mathrm{~N} / \mathrm{N}$ \\
\hline IIIZw54 & 03:41:18.729 & $+15: 24: 13.91$ & 0.0311 & $1.91 \pm 0.58$ & $1.18 \pm 0.08$ & $1.45 \pm 0.26$ & $4.57 \pm 0.56$ & Y/W \\
\hline A 1367 & $11: 44: 44.501$ & $+19: 43: 55.82$ & 0.0216 & $1.76 \pm 0.27$ & $2.11 \pm 0.14$ & $2.07 \pm 0.07$ & $4.35 \pm 0.74$ & $\mathrm{~N} / \mathrm{N}$ \\
\hline MKW4 & $12: 04: 27.660$ & $+01: 53: 41.50$ & 0.0200 & $0.50 \pm 0.14$ & $0.58 \pm 0.04$ & $0.47 \pm 0.02$ & $1.16 \pm 0.22$ & $\mathrm{Y} / \mathrm{S}$ \\
\hline ZwCl1215 & $12: 17: 40.637$ & $+03: 39: 29.66$ & 0.0750 & $4.93 \pm 0.98$ & $4.34 \pm 0.28$ & $6.10 \pm 0.29$ & $7.05 \pm 0.83$ & $\mathrm{Y} / \mathrm{N}$ \\
\hline A1650 & $12: 58: 41.885$ & $-01: 45: 32.91$ & 0.0845 & $3.44 \pm 0.66$ & $4.28 \pm 0.27$ & $5.09 \pm 0.73$ & $7.47 \pm 1.13$ & Y/W \\
\hline Coma & $12: 59: 45.341$ & $+27: 57: 05.63$ & 0.0232 & $6.55 \pm 0.79$ & $6.21 \pm 0.40$ & $8.42 \pm 0.63$ & $13.14 \pm 1.80$ & $\mathrm{~N} / \mathrm{N}$ \\
\hline A1795 & $13: 48: 52.790$ & $+26: 35: 34.36$ & 0.0616 & $3.41 \pm 0.63$ & $4.46 \pm 0.29$ & $5.11 \pm 0.14$ & $6.21 \pm 0.98$ & $\mathrm{Y} / \mathrm{S}$ \\
\hline MKW8 & $14: 40: 42.150$ & $+03: 28: 17.87$ & 0.0270 & $0.62 \pm 0.12$ & $1.10 \pm 0.07$ & $0.80 \pm 0.12$ & $1.61 \pm 0.23$ & $\mathrm{~N} / \mathrm{N}$ \\
\hline A2029 & $15: 10: 55.990$ & $+05: 44: 33.64$ & 0.0767 & $14.70 \pm 2.61$ & $6.82 \pm 0.44$ & $13.35 \pm 0.53$ & $9.59 \pm 1.11$ & $\mathrm{Y} / \mathrm{S}$ \\
\hline A2052 & $15: 16: 44.411$ & $+07: 01: 12.57$ & 0.0348 & $1.39 \pm 0.28$ & $2.03 \pm 0.13$ & $1.86 \pm 0.10$ & $3.53 \pm 0.40$ & $\mathrm{Y} / \mathrm{S}$ \\
\hline MKW3S & $15: 21: 50.277$ & $+07: 42: 11.77$ & 0.0450 & $1.45 \pm 0.34$ & $2.29 \pm 0.15$ & $2.13 \pm 0.09$ & $3.90 \pm 0.43$ & $\mathrm{Y} / \mathrm{S}$ \\
\hline A2065 & $15: 22: 29.082$ & $+27: 43: 14.39$ & 0.0721 & $11.18 \pm 1.78$ & $3.35 \pm 0.22$ & $7.66 \pm 1.44$ & $7.32 \pm 0.75$ & $\mathrm{~N} / \mathrm{W}$ \\
\hline A2142 & $15: 58: 19.776$ & $+27: 14: 00.96$ & 0.0899 & $7.36 \pm 1.25$ & $10.26 \pm 0.66$ & $13.76 \pm 0.73$ & $8.42 \pm 0.77$ & $\mathrm{Y} / \mathrm{W}$ \\
\hline A2147 & 16:02:16.305 & $+15: 58: 18.46$ & 0.0351 & $4.44 \pm 0.67$ & $3.63 \pm 0.23$ & $5.04 \pm 0.53$ & $6.84 \pm 0.90$ & $\mathrm{~N} / \mathrm{N}$ \\
\hline A2199 & $16: 28: 37.126$ & $+39: 32: 53.29$ & 0.0302 & $2.69 \pm 0.42$ & $2.64 \pm 0.17$ & $2.97 \pm 0.30$ & $4.76 \pm 0.50$ & $\mathrm{Y} / \mathrm{S}$ \\
\hline A2255 & $17: 12: 54.538$ & $+64: 03: 51.46$ & 0.0800 & $7.13 \pm 1.38$ & $4.08 \pm 0.26$ & $7.11 \pm 0.33$ & $6.74 \pm 0.97$ & $\mathrm{~N} / \mathrm{N}$ \\
\hline A2589 & $23: 23: 56.772$ & $+16: 46: 33.19$ & 0.0416 & $3.03 \pm 0.75$ & $1.88 \pm 0.12$ & $2.54 \pm 0.17$ & $5.12 \pm 0.56$ & Y/W \\
\hline
\end{tabular}

Notes. The cluster mass, $M_{500, M-M_{\mathrm{gas}}}$, is derived from the $M_{500}-M_{\mathrm{gas}, 500}$ relation, and only used for comparison with the cluster mass, $M_{500}$, derived from the "harmonic" velocity dispersion. "S", "W", and "N" denote strong cool-core, weak cool-core, and noncool-core clusters.

In our original paper, we inadvertently tabulated the stellar masses from a Kroupa (2001) initial mass function in Table 1, which are not consistent with the remaining content of the paper. We note that this does not affect any of our results. Nevertheless, we regret this error and provide the actual stellar masses in Table 1 from a Salpeter (1955) initial mass function.
Acknowledgements. The authors would like to thank Anthony Gonzalez for pointing out this error in our manuscript.

\section{References}

Kroupa, P. 2001, MNRAS, 322, 231

Salpeter, E. E. 1955, ApJ, 121, 161

\footnotetext{
* Guest astronomer at the MPE.
} 